\title{
Genome-wide identification and comprehensive analysis of $N A C$ family genes involved in fruit development in kiwifruit (Actinidia)
}

Dongfeng Jia ${ }^{1,2}$, Zhiqiang Jiang ${ }^{1,2}$, Haihui Fu' ${ }^{1}$, Lu Chen ${ }^{1,2}$, Guanglian Liao ${ }^{1,2}$, Yanqun He ${ }^{1,2}$, Chunhui Huang ${ }^{1,2^{*}}$ and Xiaobiao $\mathrm{Xu}^{1,2^{*}}$

\begin{abstract}
Background: NAC transcription factors (TFs) are plant-specific proteins encoded by a large gene family. They play important roles in diverse biological processes, such as plant growth and development, leaf senescence, and responses to biotic or abiotic stresses. Functions of a number of NAC TFs have been identified mainly in model plants. However, very few studies on NAC TFs have been conducted in the fruit tree of kiwifruit.

Results: Genome-wide NAC genes were identified and their phylogeny, genomic structure, chromosomal location, synteny relationships, protein properties and conserved motifs were analyzed. In addition, the fruit developmental process was evaluated in a new kiwifruit cultivar of Actinidia eriantha 'Ganlu 1'. And expressions for all those NAC genes were analyzed by quantitative real-time PCR method in fruits of 'Ganlu 1' during its developmental process. Our research identified 142 NAC TFs which could be phylogenetically divided into 23 protein subfamilies. The genomic structures of those NAC genes indicated that their exons were between one and ten. Analysis of chromosomal locations suggested that 116 out of 142 NACs distributed on all the 29 kiwifruit chromosomes. In addition, genome-wide gene expression analysis showed that expressions of 125 out of 142 NAC genes could be detected in fruit samples.

Conclusion: Our comprehensive study provides novel information on NAC genes and expression patterns in kiwifruit fruit. This research would be helpful for future functional identification of NAC genes involved in kiwifruit fruit development.
\end{abstract}

Keywords: Kiwifruit, NAC transcription factor, Actinidia eriantha, Fruit development, Gene expression

\section{Background}

Kiwifruit belongs to Actinidia, which is a large genus containing more than 50 species, and China is the original center of kiwifruit [1]. Fruit of kiwifruit is called "the king of fruits" as its remarkably high vitamin C

\footnotetext{
* Correspondence: lindahch@126.com; xbxu@jxau.edu.cn

${ }^{1}$ College of Agronomy, Jiangxi Agricultural University, Nanchang 330045, Jiangxi, China

Full list of author information is available at the end of the article
}

content and rich nutritional minerals for human health. By domestication and selection from wild resources, many kiwifruit varieties have been developed, so kiwifruit has become one of the most widely cultivated fruit trees around the world [2-4]. Actinidia species are dioecious plants, and their ovary from female flower is formed by several carpels. Their fresh berry fruit usually contains a lot of seeds [5], which is helpful for successfully reproducing under complex environments. The

C C The Author(s). 2021 Open Access This article is licensed under a Creative Commons Attribution 4.0 International License, which permits use, sharing, adaptation, distribution and reproduction in any medium or format, as long as you give appropriate credit to the original author(s) and the source, provide a link to the Creative Commons licence, and indicate if changes were made. The images or other third party material in this article are included in the article's Creative Commons licence, unless indicated otherwise in a credit line to the material. If material is not included in the article's Creative Commons licence and your intended use is not permitted by statutory regulation or exceeds the permitted use, you will need to obtain permission directly from the copyright holder. To view a copy of this licence, visit http://creativecommons.org/licenses/by/4.0/ The Creative Commons Public Domain Dedication waiver (http://creativecommons.org/publicdomain/zero/1.0/) applies to the data made available in this article, unless otherwise stated in a credit line to the data. 
development of kiwifruit berry fruits starts from fertilization and ends until seed maturation [6]. It was reported that during the fruit development of $A$. chinensis 'Hort16A', many aspects of fruit morphology, growth and development are similar to that of tomato (Solanum lycopersicum) fruit, except for the delay production of autocatalytic ethylene in the ripening process when fruit begins to senesce $[6,7]$.

In most cases, gene structures can be combined with expression analysis to give an insight into gene function prediction. Usually, genes with close structures have similar functions, and identifying gene family is a useful way for functional test of genes. However, so far, very few gene families have been identified in the kiwifruit genome. Notably, the genome sequence of $A$. chinensis 'Hongyang' is available since the completion of its genome project [3]. Transcription factors (TFs) are a class of proteins that regulate the temporal and/or spatial expressions of target genes by binding to cis-regulatory elements in their promoter regions [8]. Plant TFs, including AP2, bHLH, ARF, MYC, WRKY, and NAC, are essential regulators in many biological processes [9-13]. Among those TFs, NACs play important roles in diverse developmental processes in plants, such as plant growth, lateral root formation, leaf senescence, and fruit ripening and softening [14-17].

NAC protein family is one of the largest TF families in plants. A typical NAC protein contains a conserved NAC domain at its N-terminal, which comprises about 150 amino acids, and a highly variable transcriptional regulation region at the C-terminus [18, 19]. Since the first report of NAC protein in 1996 [20], NAC protein families have been identified in several plant species, such as Arabidopsis thaliana [19], rice [21], poplar [22], grapevine [23], and Asian pear [24]. In addition, functions of a number of $N A C$ genes have been uncovered in model plants. Overexpression of AtATAF1, a NAC gene of A. thaliana, enhances plant tolerance to drought stress [25]. Overexpressing OsNAC10 in rice improves plant drought stress tolerance and grain yield [26]. PopNAC122 of poplar reduces plant height growth by reducing cell size and cell number [27]. In addition to regulatory functions in stress response and plant growth, NAC proteins also play important roles in regulating fruit development. In tomato, NAC TFs of SINAC4 and NOR-like 1 have been identified as positive regulators of tomato fruit ripening [17, 28]. And tomato SINAC1 gene can alter fruit pigmentation and fruit softening in both ethylene-dependent and abscisic acid-dependent pathways [16]. In strawberry (Fragaria chiloensis), FcNAC1 protein involves in fruit softening by regulating pectin metabolism [29]. And in cucumber (Cucumis sativus), 12 NAC genes are identified to be targets of 13 micro-RNAs and they are involved in fruit development [30].

Although NAC TFs play potential roles during stress responses and developmental processes, the specific functions of most $N A C$ genes are still poorly understood, especially for those genes in kiwifruit, an important kind of berry fruit tree. To date, no systemic analysis on $N A C$ gene family has been conducted in kiwifruit. In this study, genome-wide identification of NAC proteins was conducted in the genome of $A$. chinensis 'Hongyang'. And comprehensive studies on phylogeny, gene structure, chromosomal location, and protein properties were also performed. In addition, the developmental process of fruit was evaluated in a new kiwifruit cultivar of $A$. eriantha 'Ganlu 1'. And relative expressions of all those identified $N A C$ genes were investigated by quantitative real-time PCR (qRT-PCR) method in fruits of 'Ganlu 1' during its developmental stages. Our results would be valuable for cloning and functional identification of kiwifruit $N A C$ genes. The expressing profiling data will provide essential information for revealing regulatory mechanisms of NAC TFs on kiwifruit fruit growth and development.

\section{Results}

\section{Fruit development of $A$. eriantha 'Ganlu 1'}

As shown in Fig. 1, during the developmental process from the 20 day after flowering (DAF) to the 185 DAF of 'Ganlu 1' fruit, the fruit appearance changed obviously at different stages. Specifically, the fruit size increased rapidly during the early developmental stage (from 20 DAF to $35 \mathrm{DAF}$ ). And the seeds within outer pericarp were observed from the $50 \mathrm{DAF}$ and from then on. In this process, the seed color was yellow (50 DAF), then turned to red brown (80 DAF), and then became black (110 DAF) (Fig. 1).

In addition, the fruit, longitudinal diameter, transverse diameter and fruit shape index of fruit were analyzed. The fruit weight increased rapidly during the developmental periods from 20 DAF to 110 DAF (Fig. 2a). The fruit longitudinal diameter increased rapidly during the early developmental periods (from 20 to $35 \mathrm{DAF}$ ), then slowly increased (from 35 to $110 \mathrm{DAF}$ ), and it tended to be stable in the late developmental periods (from 110 to 185 DAF) (Fig. 2b). The fruit transverse diameter showed a different variation trend than that for longitudinal diameter. The fruit exhibited a quick increasing trend for the transverse diameter during the earlier stages from the 20 to $65 \mathrm{DAF}$, and it exhibited a second growth peak during the 95 to the 125 DAF (Fig. 2c). However, the values of fruit shape index increased significantly only from 20 to $35 \mathrm{DAF}$, then they stayed the same until to 185 DAF (Fig. 2d). 


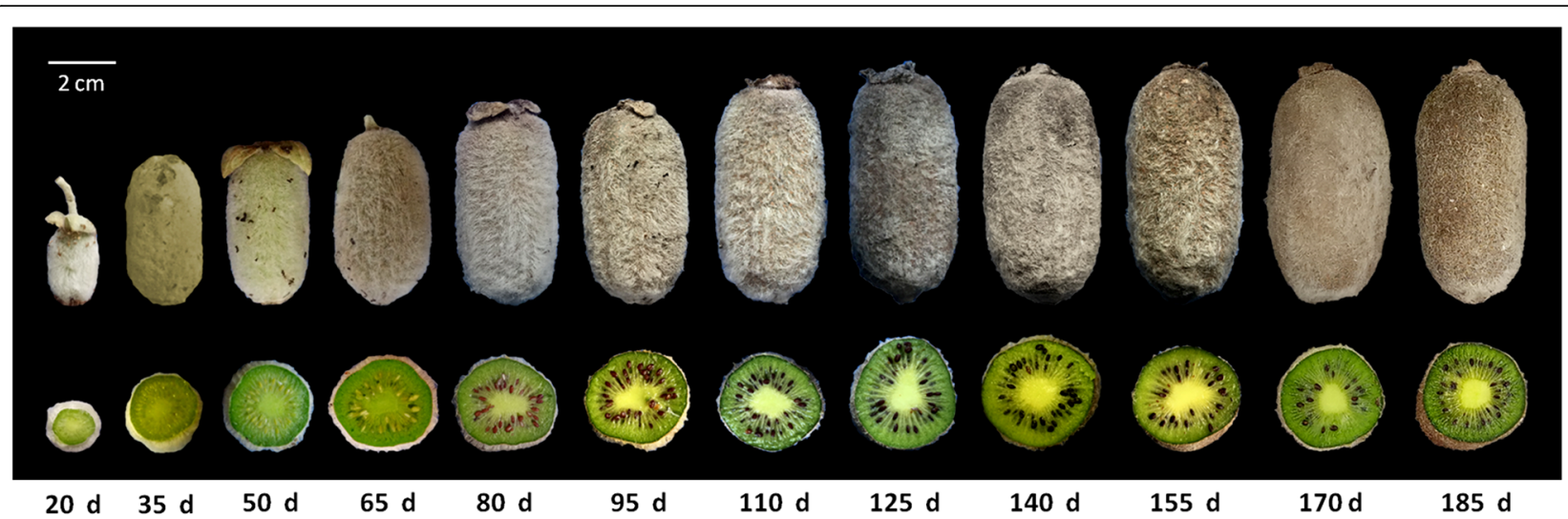

Fig. 1 Fruits of Actindia eriantha 'Ganlu 1' collected at different developmental stages. d: day after flowering

\section{Kiwifruit NAC transcription factors and their sequence} features

We identified a total of $142 N A C$ genes in the genome of $A$. chinensis 'Hongyang' and they were designated as AcNAC001 to AcNAC142, following their identifiers from the Kiwifruit Genome Database (http://kiwifruitgenome. org/) (Additional file 1). Our analysis confirmed that each of these identified AcNAC proteins contained a NAM domain (PF02365.15), a specific conserved domain of NAC TF protein family (Additional file 2). In addition, these AcNAC proteins also showed sequence similarity with Arabidopsis NAC proteins (Additional file 3).
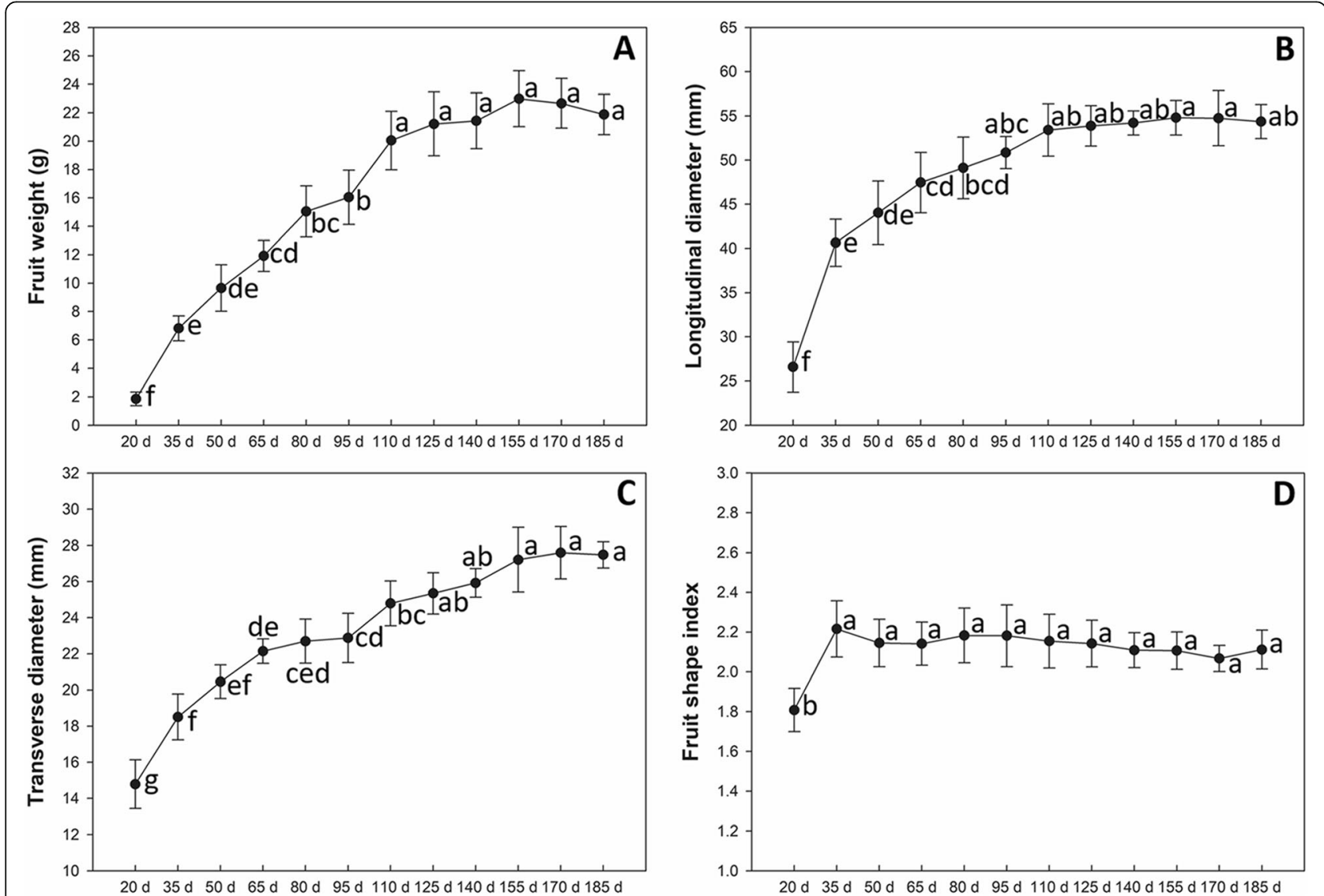

Fig. 2 Longitudinal and transverse diameters of fruit during the developmental stages in Actinidia eriantha 'Ganlu 1'. The values are presented as mean \pm standard deviation. The data were assessed by one-way ANOV and Tukey's multiple comparison tests ( $p<0.05)$. The significant differences of the data are shown by lower case letters beside the bars. d: day after flowering 
The coding sequence (CDS) lengths of these AcNAC genes were between $438 \mathrm{bp}$ and $1962 \mathrm{bp}$. And these AcNAC proteins contained 145 to 653 amino acids in length, with an average number of 328 amino acids (Additional file 4). The molecular weights were from 15, 926.35 to $74,381.42 \mathrm{Da}$, with an average value of 37 , 227.39 $\mathrm{Da}$. The predicted isoelectric points of these NAC proteins were from 4.39 to 10.24 (Additional file 4).

\section{Phylogenetic relationships of NAC proteins}

The unrooted phylogenetic tree of AcNAC proteins allowed us to separate the kiwifruit NAC protein family into 23 subfamilies. For simplicity, those subfamilies were designated alphabetically as A to W (Fig. 3). Among these subfamilies, subfamily A included 14 AcNACs members, followed by 13 in subfamily D and V, 9 in O, 8 in J, L, R and S, 7 in N and P, 6 in E and I, 5 in $\mathrm{U}$ and $\mathrm{W}, 4$ in $\mathrm{H}$ and $\mathrm{K}, 3$ in $\mathrm{B}, \mathrm{F}$ and $\mathrm{M}$. However, subfamilies of $\mathrm{C}, \mathrm{G}, \mathrm{Q}$ and $\mathrm{T}$ each contained only two NAC members (Fig. 3).
In addition, to investigate the phylogenetic relationships of NAC proteins from kiwifruit and Arabidopsis, the phylogenetic tree was constructed using all those NAC protein sequences of kiwifruit as well as that of Arabidopsis. The phylogenetic tree showed that NAC proteins of kiwifruit and Arabidopsis could be divided into 26 subfamilies, which were designated as subfamily A to $\mathrm{Z}$ (Additional file 5). In most cases, the same subfamilies from both of the two phylogenetic trees (Fig. 3 and Additional file 5) contained identical members (such as subfamilies of A, C, E, F, G, H, I, J, K, L, M, N, O, Q, $\mathrm{S}, \mathrm{T}, \mathrm{U}$ and $\mathrm{V}$ ). However, there were several exceptions, AcNAC111 from subfamily D in phylogenetic tree of kiwifruit AcNACs (Fig. 3) was clustered into subfamily B in phylogenetic tree constructed by NACs of kiwifruit and Arabidopsis (Additional file 5). The cases were similar for AcNAC058, AcNAC090, AcNAC103 and AcNAC139 (Fig. 3, Additional file 5). These above four NAC proteins along with corresponding Arabidopsis NACs formed X, Y, Z new subfamilies (Additional file 5). Besides, members of subfamilies F, I, R and V were

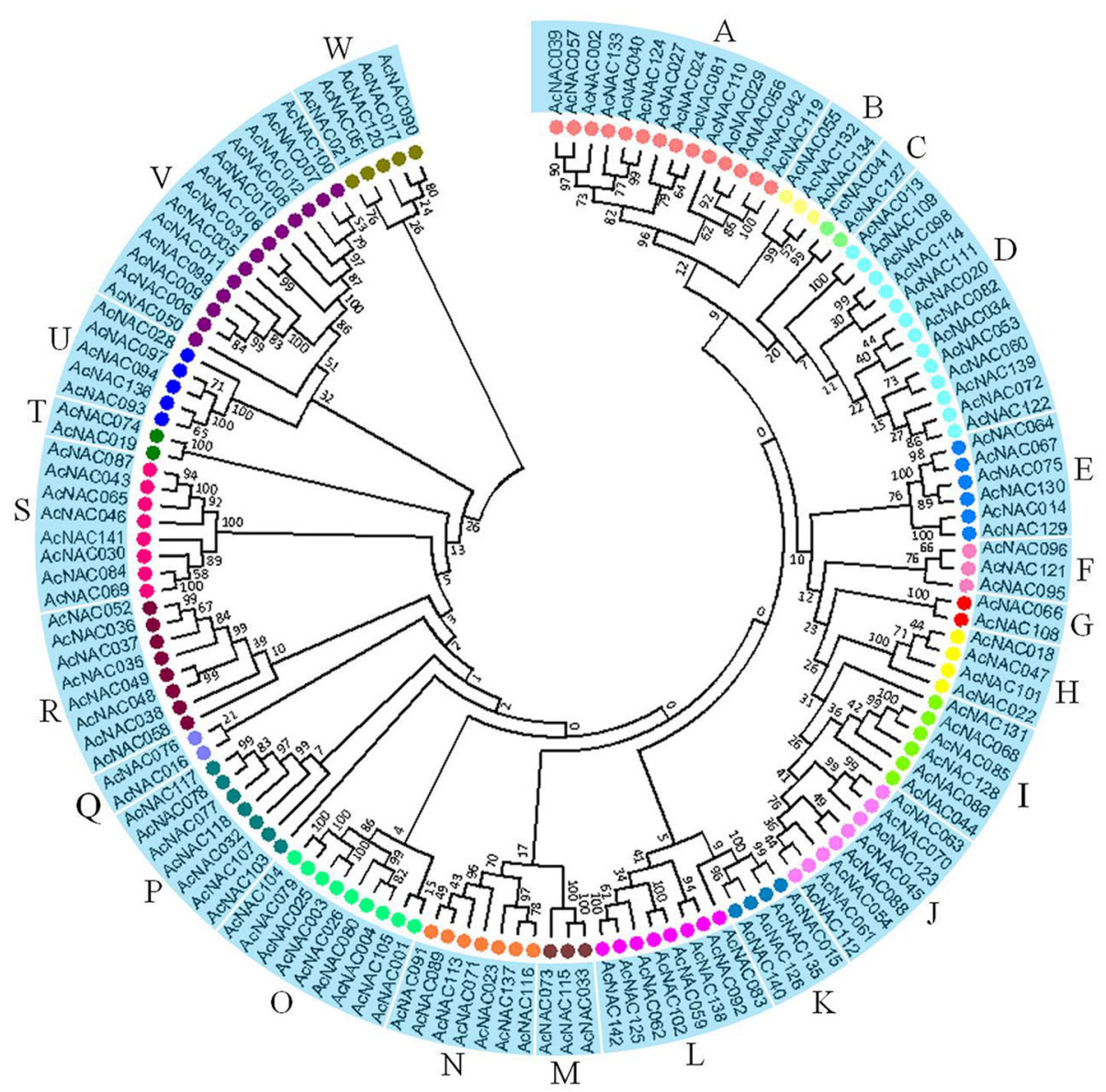

Fig. 3 Phylogenetic tree of NAC proteins in kiwifruit. The phylogenetic tree was constructed by MEGA software (version 5.05) using neighborjoining method, with 1000 bootstrap replicates. These 23 protein subfamilies are indicated by different colors, and their names are marked with upper case letters 
all from kiwifruit; conversely, NAC members of the other 22 subfamilies were from both kiwifruit and Arabidopsis (Additional file 5). These results may indicate the special functions of NAC members within those four subfamilies in kiwifruit.

\section{Genomic lengths, gene structures and conserved protein motifs}

Among these $142 A c N A C$ genes, the genomic structures were greatly different. For exon numbers, 67 AcNACs contained 3 exons (47.2\%), 25 contained 2 (14.6\%), 19 contained 4 (13.4\%), 11 contained $5(7.7 \%), 8$ contained 6 (5.6\%), 5 contained 7 (3.5\%), and the other $5 N A C$ genes (3.5\%) of AcNAC093, AcNAC136, AcNAC094, AcNAC097 and AcNAC106 each had only one exon and did not contain any intron. Besides, AcNAC038 contained 8 exons $(0.7 \%)$, and $A c N A 043$ contained the most of 10 exons and 9 introns (0.7\%) (Fig. 4, Additional file 6). For all these $A c N A C$ genes, the average numbers of exon and intron were 3.4 and 2.4, respectively (Fig. 4).

The lengths of nucleic acid bases for these AcNAC genes also varied greatly, their genomic lengths were between $555 \mathrm{bp}$ and $20,432 \mathrm{bp}$, with an average genomic length of $3592.3 \mathrm{bp}$ (Fig. 4, Additional file 6).

For those 142 NAC proteins, 10 conserved motifs were detected by employing MEME models (Fig. 4, Additional file 7). All these NAC proteins contained at least one conserved motif. And these motifs distributed at the $\mathrm{N}$-terminal for most of those NAC proteins. In general, those closely related NACs within the same subfamily had similar motif compositions. Within the AcNAC protein family, 120 out of the whole 142 members contain motif 3 , indicting their conserved domains.

\section{Chromosomal locations and synteny relationships of AcNAC genes}

Chromosomal locations of 116 out of these 142 AcNAC genes was acquired from the Kiwifruit Genome Database (Additional file 8). The 116 AcNACs were distributed along all the 29 kiwifruit chromosomes (chrs). The distribution was neither equal nor random. They were oriented either forward (+ strand) or in reverse (- strand). The numbers of $A c N A C$ genes for each individual chr were from 1 (Chr10) to 8 (Chr26), and the average numbers of $A c N A C$ genes on one chr were 4 (Fig. 5).

For distribution of $A c N A C$ genes on these chromosomes, 62 out of the 116 AcNAC genes (53.4\%) distributed on forward strands (+ strand), and 54 out of the 116 AcNAC genes (46.6\%) distributed on reverse strands (- strand) (Fig. 5, Additional file 8). However, chromosomal location information of the rest 26 AcNAC genes could not be available from the Kiwifruit Genome Database.
In addition, according to the duplicated blocks in the A. chinensis 'Hongyang' genome, 49 gene pairs with synteny relationships were identified (Fig. 5), and their genomic block information was shown in Additional file 9.

\section{Expression patterns of NAC genes in fruit during its developmental process}

The relative expression levels of all these 142 NAC genes were analyzed in fruit at different developmental stages by qRT-PCR method. The expression levels for corresponding genes of AcNACs (AcNAC001 to AcNAC142) in A. eriantha 'Ganlu 1' (AeNAC001 to AeNAC142) were analyzed. The data of expression patterns of 142 AeNAC genes were shown in Additional file 10. Among these genes, 74 NACs persistently expressed in fruit during the whole developmental process, and 51 AeNACs expressed in partial fruit samples at certain developmental stages. However, expressions of 17 AeNAC genes were not detected at any stages in fruit of $A$. eriantha 'Ganlu 1' during the whole developmental process (Fig. 6, Additional file 10).

Among the 74 AeNAC genes expressed in all the fruit samples, the expression patterns were also greatly different. Compared to the control (20 DAF), 28 AeNACs showed down-regulation patterns at some stages, and 9 $A e N A C$ s showed up-regulation patterns at some stages. Besides, 28 AeNACs showed either down- or upregulation patterns at certain stages. In addition, 6 AeNACs (AeNAC037, AeNAC054, AeNAC061, AeNAC062, AeNAC096 and AeNAC118) exhibited higher expression levels in all the fruit samples during the developmental process comparing with the control. On the contrary, 4 AeNACs (AeNAC014, AeNAC046, AeNAC103 and $A e N A C 139)$ showed decreased expression levels at each stage comparing with the control. Interestingly, the expression level of only one AeNAC gene, AeNAC087, did not change during the whole fruit developmental process (Fig. 6, Additional file 10). Among these different subfamilies, all gene members from subfamily $\mathrm{H}$ (AeNAC018, AeNAC047, AeNAC102, and AeNAC022) showed a decreased expression trend, these results may indicate their similar and negative roles in fruit development. However, within subfamilies $\mathrm{J}$ or $\mathrm{O}$, some members showed notable different expression features, this also suggested $N A C$ genes with similar structures may function differently. In short, those positive or negative relations between $N A C$ expressions and fruit development may suggest their possible roles in regulating fruit development in kiwifruit, although they may act in a complex and diverse manner.

The weighted gene co-expression network analysis (WGCNA) showed that two modules (turquoise and grey, respectively) were identified in the 142 AeNAC genes (Fig. 7a). The module-trait relationships showed that the module turquoise was closed related with the 


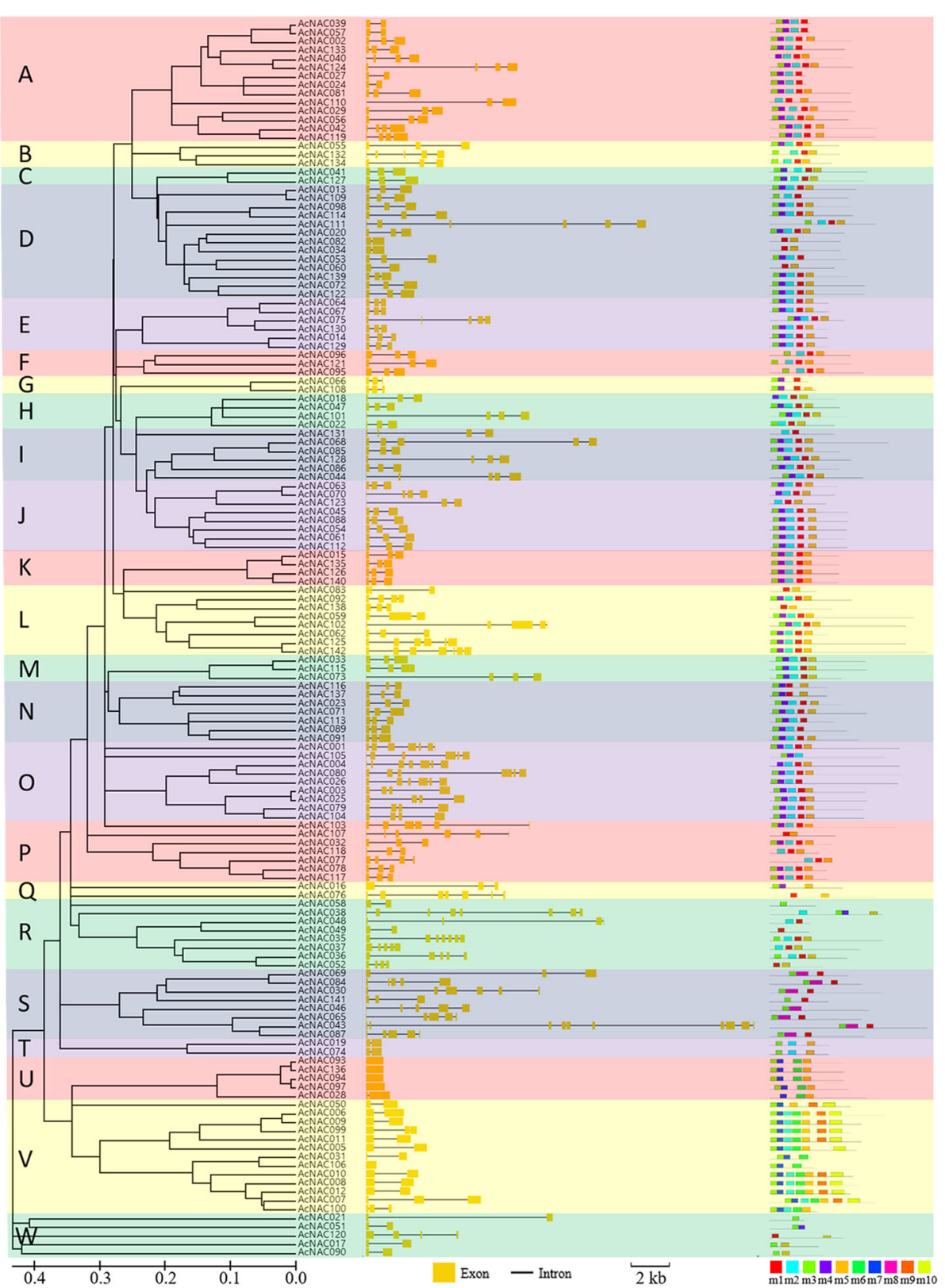

Fig. 4 Genomic structures of ACNAC genes and conserved motifs of ACNAC proteins. The yellow block and black line on the right represent exon and intron, respectively (middle). These subfamilies of NAC proteins are indicated by different colorful blocks and their names are marked with upper case letters on the left (left). These blocks with different colors show the names of protein motifs (right)

middle and late fruit developmental stages, and the module grey was mainly related with the early developmental stages (Fig. 7b). In addition, the network relationships of the NAC genes identified in module turquoise and module grey were shown in Additional file 11 and Additional file 12, respectively.

\section{Discussion}

In higher plants, fruit development is a sophisticated process, which is controlled by a number of biological and molecular mechanisms and is affected by environmental factors [31]. Understanding the regulatory pathways related to fruit development and ripening is very important for fruit quality improvement. Transcriptional regulation is an efficient way for controlling fruit development and ripening. Among those multiple TF genes, many NAC genes have been characterized to play essential roles in regulating fruit development. For example, in tomato, SINACO33 and SINACO64 control fruit development via modulating expressions of ethylene 


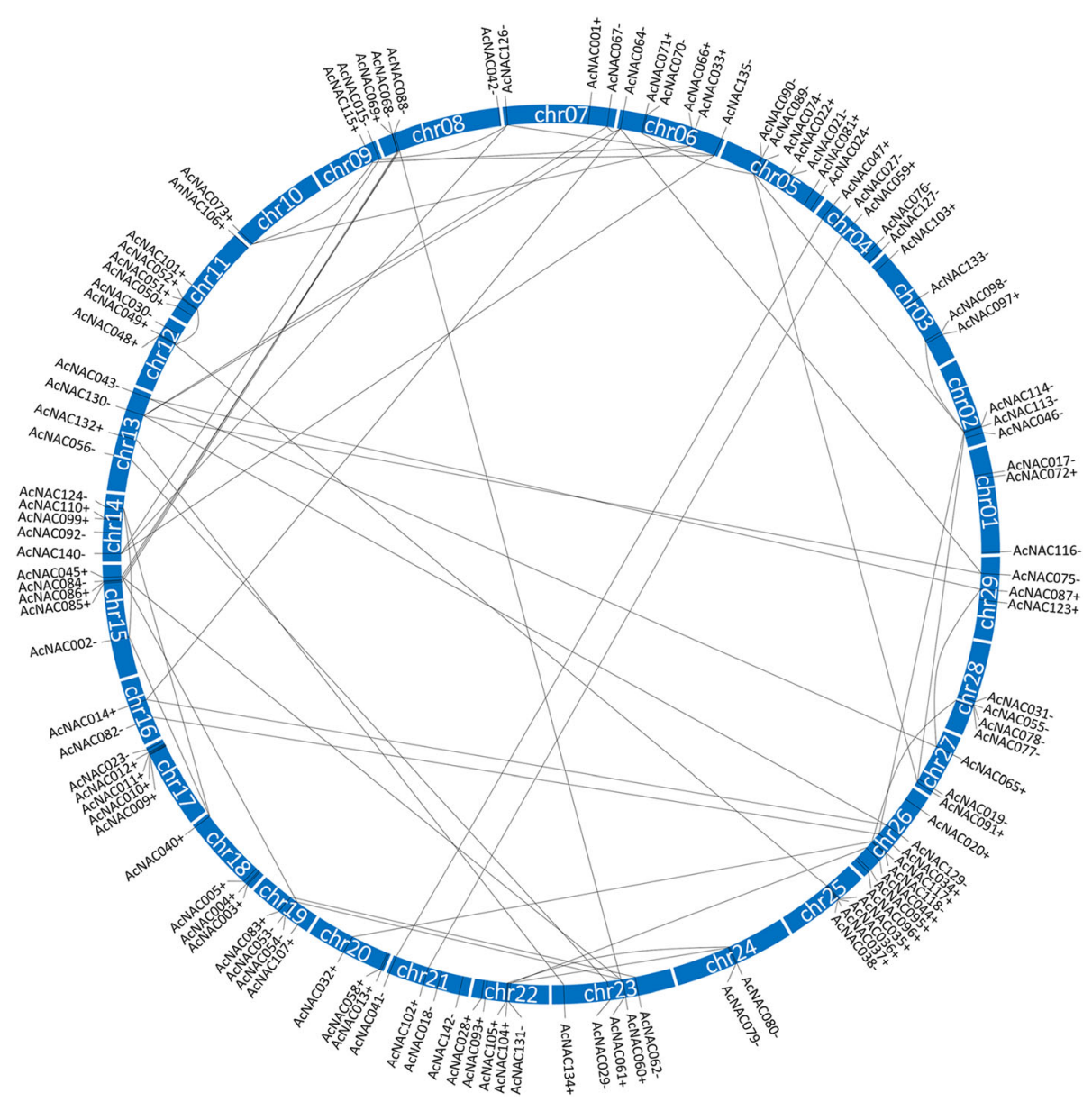

Fig. 5 Chromosomal distributions and synteny relationships of ACNAC genes in the genome of Actinidia chinensis. The kiwifruit NAC genes are marked in the vertical directions of chromosomes. " + " and "-" represent the forward strand and reverse strand, respectively. The gene pairs with synteny relationships are linked by black lines

biosynthesis and cell wall metabolism related genes [17, $28]$. Notably, little is known about the regulatory roles of $N A C$ genes in kiwifruit fruit development.

NAC protein family is a large plant-specific TF family. Since the first report of NAC [20], many studies have been conducted on NAC families in different plant species. For example, 105 NAC proteins have been identified in Arabidopsis [19], 140 in rice [21], 163 in poplar [22], 74 in grapevine [23], and 185 in Asian pear [24]. The large number of $N A C$ genes in various plant species indicates that they probably have extensively expanded in their evolutionary processes. To date, no systematic studies have been conducted on NAC family in the kiwifruit genome. Here, we identified a total of 142 kiwifruit NAC proteins based on its whole genome data. These NAC proteins were phylogenetically clustered into 23 subfamilies. Notably, NAC members of four subfamilies of $\mathrm{F}, \mathrm{I}, \mathrm{R}$ and $\mathrm{V}$ were all from kiwifruit (Additional file 5), suggesting that these genes may have a different ancestor with that of Arabidopsis during the evolutionary processes. Interestingly, the similar cases were also found in NAC gene family of tomato [32]. Hence, we speculated that those kiwifruit-specific $N A C$ genes may have specialized roles in kiwifruit plant.

In the present study, each AcNAC protein contained a conserved NAM domain (PF02365.15), a specific feature of NAC TF proteins [18]. By analyzing the chromosomal locations of AcNAC genes, we found that 116 AcNACs distributed on all the 29 kiwifruit chrs. Among them, some $A c N A C$ genes showed "clustered" distribution patterns on certain chrs, such as, AcNAC085, AcNAC086, AcNAC084 on Chr15, AcNAC009, AcNAC010, AcNAC011, AcNAC012 on Chr17, and AcNAC035, AcNAC036, AcNAC037, AcNAC038 on Chr25 (Fig. 5). Notably, most NAC genes within a same "gene clusters" belonged to the same subfamily, and they shared similar lengths and genomic structures, and the same conserved motifs (Fig. 3, Fig. 4). It is worth mentioning that in apple and tomato, many adjacent related genes (gene clusters) on the same chr also displayed similar 


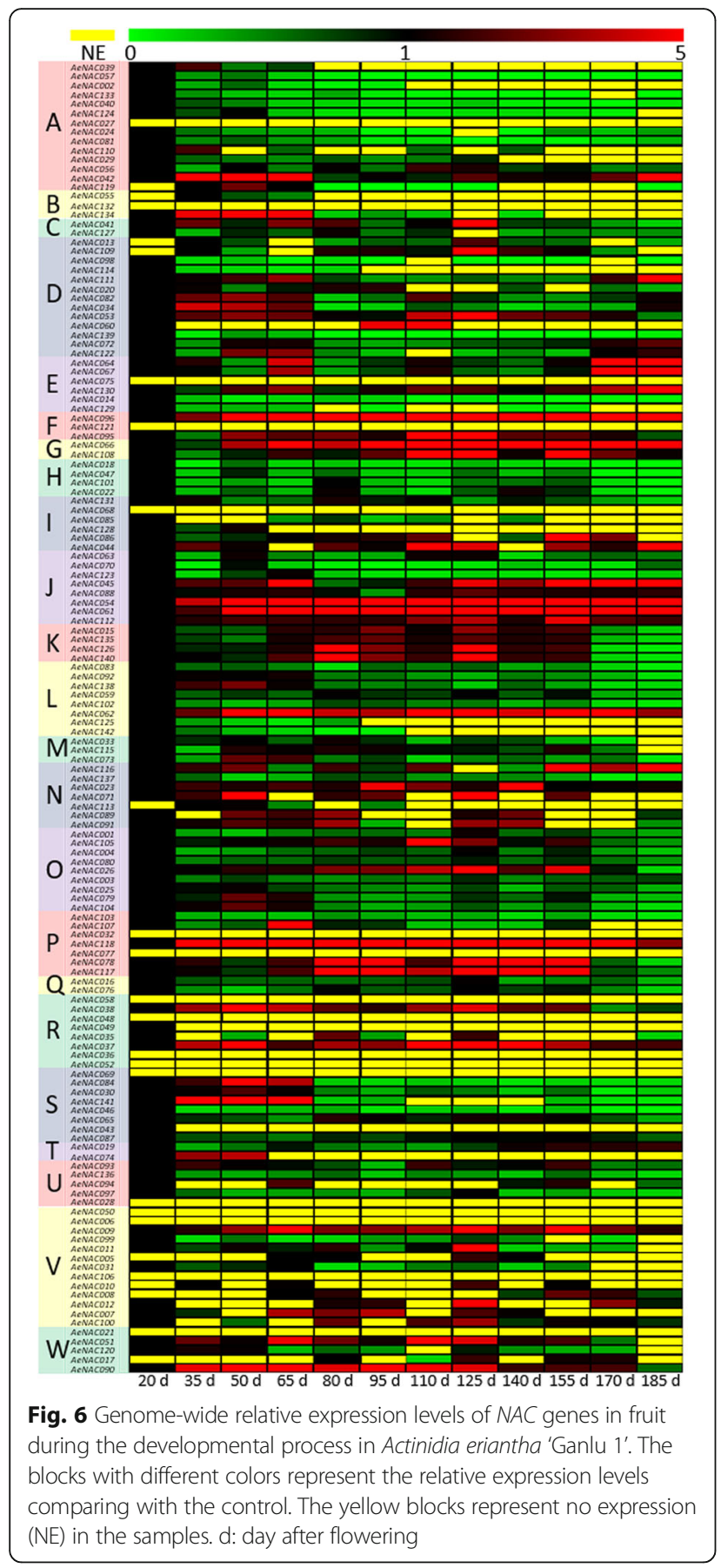

structures. And they were reported to be produced by tandem duplication events as a result of genome-wide duplication [33, 34]. Similarly, in the evolutionary process of kiwifruit genome, distributions of NBS-LRR genes indicate that tandem duplication events also happened [3]. In addition, the large number of paralogous $N A C$ gene pairs derived from genomic blocks indicated the segmentally duplicated events also happened in the genome evolution process. Therefore, we assume that the large number of $N A C$ genes may be partially caused by the tandem duplication and segmental duplication events during the evolutionary process of kiwifruit genome, which may also have important impacts on the expanding of other genes in kiwifruit genome.

To confirm NAC genes are closed involved in fruit development, genome-wide expressions of all those 142 NAC genes were examined in the developmental fruit in the new kiwifruit cultivar of $A$. eriantha 'Ganlu 1'. Particularly, three NAC genes, AeNAC054, AeNAC061, and AeNAC118 showed constitutive high expression levels during the fruit development. In addition, these $N A C$ genes were also identified as hub genes by using WGCNA. Overall, those positive correlations between fruit development and gene expressions of related $N A C$ genes probably indicated their potential regulatory roles during kiwifruit fruit growth and development. Furthermore, some NAC proteins have been reported to be involved in fruit development as well as plant growth. SINAC4 and NOR-like 1 were proved to be positive regulators of fruit ripening in tomato $[17,28]$. In banana (Musa acuminata), four NAC proteins (MaNAC016, MaNAC083, MaNAC094, and MaNAC095) were proposed to function in pulp-ripening process based on gene expression analysis [35]. FcNAC1 participates fruit ripening development in strawberry (Fragaria chiloensis) via activating $F c P L$, which is involved in fruit cell wall remodeling [36]. Arabidopsis NAC genes of AtNAP and JUB1 positively regulate fruit senescence and plant longevity [37, 38]. In addition, in woody plant apple (Malus domestica) and poplar (Рориlus trichocarpa), two NAC genes, MdNAC1 and Pop$N A C 122$, were reported to reduce plant growth in overexpression transgenic plants [27, 39]. Nevertheless, more genetic evidence is required for further understanding the functions of NAC genes in kiwifruit growth and development.

\section{Conclusion}

In this study, genome-wide identification and expression analysis of $N A C$ genes in the kiwifruit genome were performed for the first time. Although some NAC genes in other plants have been reported to be widely involved in regulating plant growth and stress responses [40, 41], the study on NAC genes in kiwifruit is still in its infancy. Our work provided crucial candidate $N A C$ genes involved in fruit growth and development. Our study also has practical value for developing early-maturing kiwifruit varieties to meet market requirements. However, further investigations still should be done to clarify the precise mechanisms of related NAC genes in regulation of fruit growth and development in kiwifruit, as well as in other fruit trees.

\section{Methods}

Plant materials and fruit development

Fruit trees of $A$. eriantha 'Ganlu 1' were planted at the Germplasm Resources Nursery of Institute of Kiwifruit, 
Cluster Dendrogram
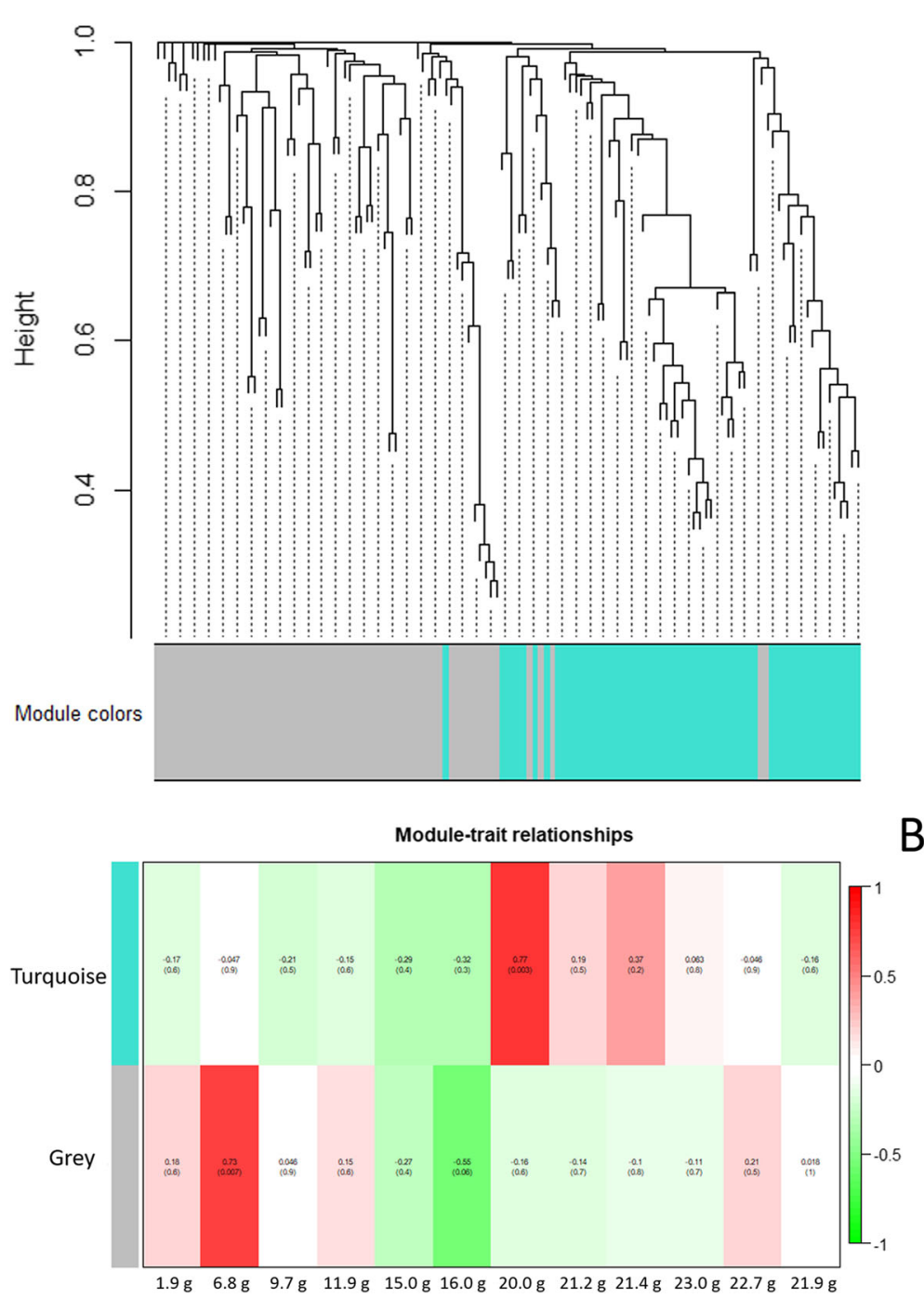

A

B expression network analysis (a). Each leaf in the tree shows one AeNAC gene. The branches constitute two modules labeled by turquoise and grey colors, respectively. $\mathbf{b}$, Module-developmental stages association. The color of each block indicates the correlation coefficient between the module and the different developmental stages. The correlation coefficient R (upper) and P-value (lower) in each block are indicated by upper number and lower number, respectively

Fengxin County $\left(28^{\circ} 70^{\prime} \mathrm{N}, 115^{\circ} 38^{\prime} \mathrm{E}\right)$, Jiangxi Province, China. Fruit samples were collected on the 20, 35, 50, 65, $80,95,110,125,140,155,170$ and 185 DAF, respectively, during the developmental process (Fig. 1). The outer pericarp without seeds was cut into sections and rapidly frozen in liquid nitrogen, and stored at $-80^{\circ} \mathrm{C}$ until use.

To evaluate fruit size during the developmental process, longitudinal diameter and transverse diameter of fruits collected at each stage were measured. At least 8 fruits were measured for each experiment. The data were assessed by one-way ANOV and Tukey's multiple comparison tests $(p<0.05)$ using SPSS Statistics software (version 20).

\section{Genome-wide identification of NAC protein family}

Genome sequence data of $A$. chinensis 'Hongyang' were downloaded from the Kiwifruit Genome Database [3] and they were used for identifying members of NAC TF protein family. Firstly, we performed a local BLASTP program search by BioEdit software [42] against all these genome protein sequences of kiwifruit, using 105 Arabidopsis NAC protein sequences (Additional file 3) downloaded from the Arabidopsis Information Resource site (https://www.arabidopsis.org/) as queries with an expectation value (E-value) < 1.0. Secondly, the predicted NAC protein sequences of $A$. chinensis were also downloaded from the Plant Transcription Factor Database (http:// 
planttfdb.cbi.pku.edu.cn/) to collect entire candidates of NAC proteins in kiwifruit. All candidates collected by the above two methods were used for further identification. Three steps were taken to verify all these candidates. First, hidden Markov models (HMMs) of the Pfam 32.0 database (http://pfam.xfam.org/) were used to identify the NAM domain (PF02365.15) for each protein sequence under an E-value $<1.0$ [43]. Second, conserved domains of the National Center for Biotechnology Information (https://blast.ncbi.nlm.nih.gov/) were also exploited to confirm the existence of NAM domains for all identified sequences [44]. Those protein sequences that do not contain NAM domain or have ambiguous domain belonging to other protein family were excluded. Third, we carefully examined all these candidate sequences and manually excluded protein sequences with a length less than 130 amino acids. Finally, these remaining protein sequences were regarded as NAC TFs of $A$. chinensis (AcNACs) and they were used for subsequent evaluation.

LaserGene software (version 7.1) was used to analyze the predicted isoeletric point and molecular weight of AcNAC proteins. And DNAMAN software (version 6.0) was used for the multiple sequence alignment analysis of AcNAC proteins and their corresponding similar sequences from Arabidopsis.

\section{Phylogenetic analysis of kiwifruit NAC proteins}

To analyze the phylogenetic relationships of the AcNAC proteins, multiple sequence alignments were performed among these AcNAC proteins using MUSCLE method with default parameters [45]. An unrooted phylogenetic tree was constructed by MEGA software (version 5.05) using neighbor-joining method, with 1000 bootstrap replicates [46]. These same methods were also used for constructing phylogenetic tree of NAC proteins from kiwifruit and Arabidopsis.

\section{Analysis of structures and conserved motifs of proteins} Information on genomic chromosomal location and CDS of each AcNAC gene was downloaded from the Kiwifruit Genome Database. The genomic length and introns/exons organization for each individual AcNAC gene were displayed by the Gene Structure Display Server (version 2.0) (http://gsds.cbi.pku.edu.cn/) [47]. Details of genomic length and exons for those $A c N A C$ genes (AcNACO01 to AcNAC142) were presented in Additional file 6.

The conserved motifs in those 142 AcNAC protein sequences were elucidated using online MEME tool (version 5.1.1; http://meme-suite.org/). Zero or One Occurrence Per Sequence was used and motif width was set between 19 and 50 residues.

\section{Chromosomal distribution analysis and gene synteny analyses}

The relative locations of $A c N A C$ genes on different chrs as well as their chromosomal directions (+ strand or strand) were also analyzed. The detailed information was shown in Additional file 8.

To explore the synteny relationships of NAC genes in the genome of $A$. chinensis 'Hongyang', the information of paired homologous $N A C$ genes with common genomic blocks was obtained from the Kiwifruit Genome Database and was used to show the synteny relationships between NAC genes. The detailed information was shown in Additional file 9.

\section{Genome-wide expression analysis of NAC genes in developmental fruit and their gene co-expression network analysis}

Total RNA of frozen fruit samples was extracted using a RNA isolation Kit (Huayueyang, Beijing, China) with a step of removing genomic DNA by DNase I. First-strand cDNA was synthesized with a PrimeScript Reverse Transcriptase Kit (Takara; Dalian, China).

To clarify the expression patterns of NAC gene in developmental fruits of $A$. eriantha 'Ganlu 1', the relative expressions of all those 142 AeNAC genes were analyzed by qRT-PCR method using a LightCycler 480 Real-Time PCR machine (Applied Biosystems, Waltham, MA, USA). The qRT-PCR analysis was performed with specific primers, using $A c N A C$ sequences as templates (Additional file 13). The results were calculated with $2^{-\Delta \Delta C T}$ method [48]. The kiwifruit Actin gene (GenBank accession number: FG515334.1) (Additional file 13) was used as the internal control to standardize the cDNA samples. Each experiment was conducted with three replicates.

To visually describe the expressing results, compared with the control (the first day when expression detected, mostly the $20 \mathrm{DAF}$ ), those relative expression levels with a fold change $>2.0$ or $<0.5$ were designated as increasing or decreasing expression, respectively. Meanwhile, when fold change of relative expression levels were between 0.5 and 2.0, those expressing results were defined as unchanged.

In addition, to identify the correlations among all those $A e N A C$ genes, WGCNA was conducted using the average relative expression data (Additional file 10) of the 142 NAC genes with an R package [49]. The modules and their corresponding gene network data were obtained according to previous method [44] and those data were referred to identify hub genes. The moduledevelopmental traits association analysis was also conducted [50]. The key R script parameters (optimum beta value and co-expression matrix) used for WGCNA were shown in Additional file 14. 


\section{Supplementary Information}

The online version contains supplementary material available at https://doi. org/10.1186/s12870-020-02798-2

Additional file 1. ACNAC genes and their predicted coding or protein sequences identified in the genome of Actinidia chinensis 'Hongyang'.

Additional file 2. ACNAC proteins and their conserved NAM domains identified by hidden Markov models.

Additional file 3. Similarity of kiwifruit NAC proteins and the corresponding NAC proteins from Arabidopsis.

Additional file 4. Primary information of ACNAC proteins and the lengths of their corresponding coding sequences (CDS).

Additional file 5. Phylogenetic tree of NAC proteins from kiwifruit and Arabidopsis. The phylogenetic tree was constructed by MEGA software (version 5.05) using neighbor-joining method, with 1000 bootstrap replicates. Subfamilies of NAC proteins are indicated by different colors, and their names are marked with upper case letters.

Additional file 6. Genomic length, exon number and length of ACNAC genes.

Additional file 7. Amino acid residue sequences of conserved motifs identified in the ACNAC proteins.

Additional file 8. Chromosomal locations and distribution directions of ACNAC genes.

Additional file 9. Synteny relationships and their genomic blocks of ACNAC gene pairs.

Additional file 10. Relative expression data of 142 NAC genes in fruit during the developmental process in Actinidia eriantha 'Ganlu 1'.

Additional file 11. The enriched cluster information of expressing comparisons for the input genes from turquoise module identified by weighted gene co-expression network analysis.

Additional file 12. The enriched cluster information of expressing comparisons for the input genes from grey module identified by weighted gene co-expression network analysis.

Additional file $\mathbf{1 3}$ Primer sequences used for relative expression analysis of AeNAC genes with quantitative real-time PCR methods.

Additional file 14. The key R script parameters of optimum beta value and co-expression matrix used for weighted gene co-expression network analysis.

\section{Abbreviations}

CDS: Coding sequence; chr: Chromosome; HMM: Hidden Markov models; NE: No expression; qRT-PCR: Quantitative real-time PCR; TF: Transcription factor; WGCNA: Weighted gene co-expression network analysis

\section{Acknowledgments}

We are grateful to Dr. Mingjun Li (Northwest A \& F University, Yangling, China) for his intellectual input in the study. We are grateful to Dr. Tao Zhao (Northwest A \& F University, Yangling, China) for his important suggestions in gene expressing analysis. We are also grateful to Dr. Huibin Han (Jiangxi Agricultural University, Nanchang, China) for his help in revising the paper.

\section{Authors' contributions}

D.J. and L.C. performed most of the experiments. D.J. wrote the paper. Z.J. and Y.H. assisted with the RNA isolation. H.F. conducted the weighted gene co-expression network analysis. G.L. helped with the gene expression analysis. X.X., C.H., and D.J. provided the financial support. All authors have read and approved the manuscript.

\section{Funding}

This study was supported by National Natural Science Foundation of China (31760559, 31760567 and 31960588), Key Research and Development Plan of Jiangxi Science and Technology Department (20192ACB60002), and Start-up Fund for Doctors from Jiangxi Agricultural University (9232307422). The funding bodies were not involved in the study design, collection, analysis, interpretation of data, and in writing the manuscript.

\section{Availability of data and materials}

The datasets supporting the conclusions of this article are available online from the Kiwifruit Genome Database, the Arabidopsis Information Resource site, the GenBank database, or included within the article additional files.

Ethics approval and consent to participate

Not applicable.

\section{Consent for publication}

All authors agreed to publish.

\section{Competing interests}

The authors declare that they have no competing interests.

\section{Author details}

${ }^{1}$ College of Agronomy, Jiangxi Agricultural University, Nanchang 330045, Jiangxi, China. ${ }^{2}$ Institute of Kiwifruit, Jiangxi Agricultural University, Nanchang 330045, Jiangxi, China.

Received: 30 June 2020 Accepted: 16 December 2020

Published online: 15 January 2021

\section{References}

1. Ferguson AR, Huang $\mathrm{H}$. Genetic resources of kiwifruit: domestication and breeding. In: Janick J, editor. Horticultural Reviews. Hoboken: Wiley; 2007.

2. Laing WA, Bulley S, Wright M, Cooney J, Jensen D, Barraclough D, MacRae E. A highly specific L-galactose-1-phosphate phosphatase on the path to ascorbate biosynthesis. Proc Natl Acad Sci U S A. 2004;101:16976-81.

3. Huang S, Ding J, Deng D, Tang W, Sun H, Liu D, Zhang L, Niu X, Zhang X, Meng M, Yu J, Liu J, Han Y, Shi W, Zhang D, Cao S, Wei Z, Cui Y, Xia Y, Zeng H, Bao K, Lin L, Min Y, Zhang H, Miao M, Tang X, Zhu Y, Sui Y, Li G, Sun H, Yue J, Sun J, Liu F, Zhou L, Lei L, Zheng X, Liu M, Huang L, Song J, Xu C, Li J, Ye K, Zhong S, Lu BR, He G, Xiao F, Wang HL, Zheng H, Fei Z, Liu Y. Draft genome of the kiwifruit Actinidia chinensis. Nat Commun. 2013;4:2640.

4. Park YS, Im MH, Ham KS, Kang SG, Park YK, Namiesnik J, Leontowicz H, Leontowicz M, Katrich E, Gorinstein S. Nutritional and pharmaceutical properties of bioactive compounds in organic and conventional growing kiwifruit. Plant Foods Hum Nutr. 2013;68:57-64.

5. Ferguson AR. Kiwifruit: a botanical review. In: Janick J, editor. Horticultural Reviews. Westport: AVI Publishing Company, Inc; 1984.

6. Richardson AC, Boldingh HL, McAtee PA, Gunaseelan K, Luo Z, Atkinson RG, David KM, Burdon JN, Schaffer RJ. Fruit development of the diploid kiwifruit, Actinidia chinensis 'Hort16A'. BMC Plant Biol. 2011;11:182.

7. Giovannoni JJ. Genetic regulation of fruit development and ripening. Plant Cell. 2004;16:S170-80.

8. Guo Y, Gan S. AtNAP, a NAC family transcription factor, has an important role in leaf senescence. Plant J. 2006;46:601-12.

9. Yamasaki K, Kigawa T, Inoue M, Watanabe S, Tateno M, Seki M, Shinozaki K, Yokoyama S. Structures and evolutionary origins of plant-specific transcription factor DNA-binding domains. Plant Physiol Biochem. 2008;46: 394-401.

10. Tisza V, Kovács L, Balogh A, Heszky L, Kiss E. Characterization of FaSPT, a SPATULA gene encoding a bHLH transcriptional factor from the nonclimacteric strawberry fruit. Plant Physiol Biochem. 2010;48:822-6.

11. Lee JM, Joung JG, McQuinn R, Chung MY, Fei Z, Tieman D, Klee H, Giovannoni JJ. Combined transcriptome, genetic diversity and metabolite profiling in tomato fruit reveals that the ethylene response factor SIERF6 plays an important role in ripening and carotenoid accumulation. Plant J. 2012;70:191-204.

12. Zhang D, Ren L, Yue JH, Shi YB, Zhuo LH, Wang L, Shen XH. RNA-Seq-based transcriptome analysis of stem development and dwarfing regulation in Agapanthus praecox ssp. orientalis (Leighton) Leighton. Gene. 2015;565:252-67.

13. Kato H, Motomura T, Komeda Y, Saito K, Kato A. Overexpression of the NAC transcription factor family gene ANACO36 results in a dwarf phenotype in Arabidopsis thaliana. J Plant Physiol. 2010;167:571-7.

14. Xie Q. Arabidopsis NAC1 transduces auxin signal downstream of TIR1 to promote lateral root development. Genes Dev. 2000;14:3024-36.

15. Olsen AN, Ernst HA, Leggio LL, Skriver K. NAC transcription factors: structurally distinct, functionally diverse. Trends Plant Sci. 2005;10:79-87. 
16. Ma N, Feng H, Meng X, Li D, Yang D, Wu C, Meng Q. Overexpression of tomato SINACl transcription factor alters fruit pigmentation and softening. BMC Plant Biol. 2014;14:351.

17. Zhu M, Chen G, Zhou S, Tu Y, Wang Y, Dong T, Hu Z. A new tomato NAC (NAM/ATAF1/2/CUC2) transcription factor, SINAC4, functions as a positive regulator of fruit ripening and carotenoid accumulation. Plant Cell Physiol. 2014;55:119-35

18. Aida M. Genes involved in organ separation in Arabidopsis: an analysis of the cup-shaped cotyledon mutant. Plant Cell. 1997;9:841-57.

19. Ooka H, Satoh K, Doi K, Nagata T, Otomo Y, Murakami K, Matsubara K, Osato N, Kawai J, Carninci P, Hayashizaki Y, Suzuki K, Kojima K, Takahara Y, Yamamoto K, Kikuchi S. Comprehensive analysis of NAC family genes in Oryza sativa and Arabidopsis thaliana. DNA Res. 2003;10:239-47.

20. Souer $E$, van Houwelingen $A$, Kloos D, Mol J, Koes R. The no apical meristem gene of petunia is required for pattern formation in embryos and flowers and is expressed at meristem and primordia boundaries. Cell. 1996;85:159-70.

21. Fang Y, You J, Xie K, Xie W, Xiong L. Systematic sequence analysis and identification of tissue-specific or stress-responsive genes of NAC transcription factor family in rice. Mol Gen Genomics. 2008;280:547-63.

22. Hu R, Qi G, Kong Y, Kong D, Gao Q, Zhou G. Comprehensive analysis of NAC domain transcription factor gene family in Populus trichocarpa. BMC Plant Biol. 2010;10:145

23. Wang $N$, Zheng $Y$, Xin H, Fang L, Li S. Comprehensive analysis of NAC domain transcription factor gene family in Vitis vinifera. Plant Cell Rep. 2013; 32:61-75.

24. Ahmad M, Yan X, Li J, Yang Q, Jamil W, Teng Y, Bai S. Genome wide identification and predicted functional analyses of NAC transcription factors in Asian pears. BMC Plant Biol. 2018;18:214.

25. Wu Y, Deng Z, Lai J, Zhang Y, Yang C, Yin B, Zhao Q, Zhang L, Li Y, Yang C, Xie Q. Dual function of Arabidopsis ATAF1 in abiotic and biotic stress responses. Cell Res. 2009;19:279.

26. Jeong JS, Kim YS, Baek KH, Jung H, Ha SH, Choi YD, Kim M, Kim JK. Rootspecific expression of OsNAC10 improves drought tolerance and grain yield in rice under field drought conditions. Plant Physiol. 2010;153:185-97.

27. Grant EH, Fujino T, Beers EP, Brunner AM. Characterization of NAC domain transcription factors implicated in control of vascular cell differentiation in Arabidopsis and Populus. Planta. 2010;232:337-52.

28. Gao Y, Wei W, Zhao X, Tan X, Fan Z, Zhang Y, Jing Y, Meng L, Zhu B, Zhu H, Chen J, Jiang CZ, Grierson D, Luo Y, Fu DQ. A NAC transcription factor, NOR-like1, is a new positive regulator of tomato fruit ripening. Hortic Res. 2018:5:75.

29. Carrasco-Orellana C, Stappung Y, Mendez-Yañez A, Allan AC, Espley RV, Plunkett BJ, Moya-Leon MA, Herrera R. Characterization of a ripeningrelated transcription factor FcNAC1 from Fragaria chiloensis fruit. Sci Rep. 2018;8: 10524.

30. Liu X, Wang T, Bartholomew E, Black K, Dong M, Zhang Y, Yang S, Cai Y, Xue S, Weng Y, Ren H. Comprehensive analysis of NAC transcription factors and their expression during fruit spine development in cucumber (Cucumis sativus L.). Hortic Res. 2018:5:31.

31. Tanou G, Minas IS, Karagiannis E, Tsikou D, Audebert S, Papadopoulou KK, Molassiotis A. The impact of sodium nitroprusside and ozone in kiwifruit ripening physiology: a combined gene and protein expression profling approach. Ann Bot. 2015;116:649-62.

32. Jin JF, Wang ZQ, He QY, Wang JY, Li PF, Xu JM, Zheng SJ, Fan W, Yang JL. Genome-wide identification and expression analysis of the NAC transcription factor family in tomato (Solanum lycopersicum) during aluminum stress. BMC Genomics. 2020;1:288.

33. Velasco R, Zharkikh A, Affourtit J, Dhingra A, Cestaro A, Kalyanaraman A, Fontana P, Bhatnagar SK, Troggio M, Pruss D, Salvi S, Pindo M, Baldi P, Castelletti S, Cavaiuolo M, Coppola G, Costa F, Cova V, Dal RA, Goremykin V, Komjanc M, Longhi S, Magnago P, Malacarne G, Malnoy M, Micheletti D, Moretto M, Perazzolli M, Si-Ammour A, Vezzulli S, Zini E, Eldredge G, Fitzgerald LM, Gutin N, Lanchbury J, Macalma T, Mitchell JT, Reid J, Wardell B, Kodira C, Chen Z, Desany B, Niazi F, Palmer M, Koepke T, Jiwan D, Schaeffer S, Krishnan V, Wu C, Chu VT, King ST, Vick J, Tao Q, Mraz A, Stormo A, Stormo K, Bogden R, Ederle D, Stella A, Vecchietti A, Kater MM, Masiero S, Lasserre P, Lespinasse Y, Allan AC, Bus V, Chagne D, Crowhurst RN, Gleave AP, Lavezzo E, Fawcett JA, Proost S, Rouze P, Sterck L, Toppo S, Lazzari B, Hellens RP, Durel CE, Gutin A, Bumgarner RE, Gardiner SE, Skolnick M, Egholm M, Van de Peer Y, Salamini F, Viola R. The genome of the domesticated apple (Malus $\times$ domestica Borkh.). Nat Genet. 2010;42:833-9.
34. The Tomato Genome Consortium. The tomato genome sequence provides insights into fleshy fruit evolution. Nature. 2012;485:635-41.

35. Li B, Fan R, Yang Q, Hu C, Sheng O, Deng G, Dong T, Li C, Peng X, Bi F, Yi G Genome-wide identification and characterization of the NAC transcription factor family in Musa Acuminata and expression analysis during fruit ripening. Int J Mol Sci. 2020;21:634.

36. Carrasco-Orellana C, Stappung Y, Mendez-Yañez A, Allan AC, Espley RV, Plunkett BJ, Moya-Leon MA, Herrera R. Characterization of a ripening-related transcription factor FcNAC1 from Fragaria chiloensis fruit. Sci Rep. 2018;8:24.

37. Kou X, Watkins CB, Gan SS. Arabidopsis AtNAP regulates fruit senescence. J Exp Bot. 2012;63:6139-47.

38. Wu A, Allu AD, Garapati P, Siddiqui H, Dortay H, Zanor Ml, Asensi-Fabado MA, Munné-Bosch S, Antonio C, Tohge T, Fernie AR, Kaufmann K, Xue GP, Mueller-Roeber B, Balazadeh S. JUNGBRUNNEN1, a reactive oxygen speciesresponsive NAC transcription factor, regulates longevity in Arabidopsis. Plant Cell. 2012;24:482-506.

39. Jia D, Gong X, Li M, Li C, Sun T, Ma F. Overexpression of a novel apple NAC transcription factor gene, MdNACl, confers the dwarf phenotype in transgenic apple (Malus domestica). Genes. 2018;9:229.

40. Kim HJ, Nam HG, Lim PO. Regulatory network of NAC transcription factors in leaf senescence. Curr Opin Plant Biol. 2016;33:48-56.

41. Nuruzzaman M, Sharoni AM, Kikuchi S. Roles of NAC transcription factors in the regulation of biotic and abiotic stress responses in plants. Front Microbiol. 2013;4:248.

42. Altschul SF, Madden TL, Schffer AA, Zhang J, Zhang Z, Miller W, Lipman DJ. Gapped BLAST and PSI-BLAST: a new generation of protein database search programs. Nucleic Acids Res. 1997;25:3389-402.

43. El-Gebali S, Mistry J, Bateman A, Eddy SR, Luciani A, Potter SC, Qureshi M, Richardson LJ, Salazar GA, Smart A, Sonnhammer ELL, Hirsh L, Paladin L, Piovesan D, Tosatto SCE, Finn RD. The Pfam protein families database in 2019. Nucleic Acids Res. 2019;D1:D427-32.

44. Marchler-Bauer A, Bo Y, Han L, He J, Lanczycki CJ, Lu S, Chitsaz F, Derbyshire MK, Geer RC, Gonzales NR, Gwadz M, Hurwitz DI, Lu F, Marchler GH, Song JS, Thanki N, Wang Z, Yamashita RA, Zhang D, Zheng C, Geer LY, Bryant SH. CDD/SPARCLE: functional classification of proteins via subfamily domain architectures. Nucleic Acids Res. 2016;45:D200-3.

45. Edgar RC. MUSCLE: multiple sequence alignment with high accuracy and high throughput. Nucleic Acids Res. 2004;32:1792-7.

46. Tamura K, Peterson D, Peterson N, Stecher G, Nei M, Kumar S. MEGA5: molecular evolutionary genetics analysis using maximum likelihood, evolutionary distance, and maximum parsimony methods. Mol Biol Evol. 2011:8:2731-9.

47. Hu B, Jin J, Guo AY, Zhang H, Luo J, Gao G. GSDS 2.0: an upgraded gene feature visualization server. Bioinformatics. 2015;31:1296-7.

48. Livak K, Schmittgen TD. Analysis of relative gene expression data using real time quantitative PCR and the $2^{-\Delta \Lambda C T}$ method. Methods. 2001;25:402-8.

49. Langfelder P, Horvath S. WGCNA: an R package for weighted correlation network analysis. BMC Bioinformatics. 2008;9:559.

50. Wu J, Fu L, Yi H. Genome-wide identification of the transcription factors involved in Citrus fruit ripening from the transcriptomes of a late-ripening sweet orange mutant and its wild type. PLoS One. 2016;11:e0154330.

\section{Publisher's Note}

Springer Nature remains neutral with regard to jurisdictional claims in published maps and institutional affiliations.

\section{Ready to submit your research? Choose BMC and benefit from:}

- fast, convenient online submission

- thorough peer review by experienced researchers in your field

- rapid publication on acceptance

- support for research data, including large and complex data types

- gold Open Access which fosters wider collaboration and increased citations

- maximum visibility for your research: over $100 \mathrm{M}$ website views per year

At BMC, research is always in progress.

Learn more biomedcentral.com/submissions 